\title{
The circumstellar environment of the star V923 Aquilae
}

\author{
M. L. Arias ${ }^{\star \star \star \star}$, L. S. Cidale ${ }^{\star \star \star \star \star \star}$, and A. E. Ringuelet ${ }^{\star \star \star}$ \\ Facultad de Ciencias Astronómicas y Geofísicas, Universidad Nacional de La Plata, Paseo del Bosque S/N, 1900 CGA La Plata, \\ Argentina
}

Received 2 October 2003 / Accepted 5 December 2003

\begin{abstract}
V923 Aquilae is a Be shell star that displays emission in $\mathrm{H} \alpha$, cyclic $V / R$ variations and variations in the intensity of the near infrared continuum. The star presents radial velocity variations arising from a superposition of a long-term cycle and an orbital motion with a period of 214.756 days (Koubský et al. 1989). To investigate the physical properties and the geometry of the circumstellar envelope of this star, we have analysed its spectra in the UV and visual range. We have selected Fe II lines to derive temperatures and location of the line-forming regions. Our results indicate that the dimensions of the circumstellar envelope vary with time and this variation correlates with the orbital period of 214.75 days. On the other hand, we determined a period of 6.8 years for $V / R$ cyclic variations in $\mathrm{H} \alpha$. This period is in accordance with the long-term cycle in the radial velocity and $\mathrm{U}$ band photometric observations. We suggest that the $V / R$ variability in the $\mathrm{H} \alpha$ line is linked to the behavior of the subsonic wind structure.
\end{abstract}

Key words. stars: emission-line, Be - stars: circumstellar matter

\section{Introduction}

V923 Aquilae (HD $183656=$ HR $7415=$ MWC 988, $\left.\alpha(2000)=19^{\mathrm{h}} 30^{\mathrm{m}} 32.9^{\mathrm{s}}, \delta(2000)=+03^{\circ} 26^{\prime} 39.7^{\prime \prime}, V=6.05\right)$ is a Be shell star that displays a B6 stellar spectrum, first studied by Harper (1937) and Bidelman (1950), whose main optical features are rotationally broadened He I lines, narrow metallic lines, $\mathrm{H}$ lines with strong sharp cores and double emission components in the first members of the Balmer series. This star exhibits spectroscopic and photometric variations on both longterm and short-term time scales. Merrill (1952) and Merrill \& Lowen (1953) found variations in the radial velocities of the shell lines as well as in the $V / R$ ratio of the double emission peak of $\mathrm{H} \alpha$ and $\mathrm{H} \beta$. They suggested that hydrogen and metallic lines varied with a period of perhaps 6.5 years and an amplitude of $60 \mathrm{~km} \mathrm{~s}^{-1}$. Later, Koubský et al. (1989) demonstrated that the observed radial velocity variation arises from a superposition of cyclic long-term velocity variations of variable amplitude and cycle length $\left(20 \leq 2 \mathrm{~K} \leq 65 \mathrm{~km} \mathrm{~s}^{-1}\right.$ and $1800 \leq P \leq 2400$ days respectively) and an orbital motion with a period of 214.75 days and an amplitude of $12.4 \mathrm{~km} \mathrm{~s}^{-1}$.

Send offprint requests to: M. L. Arias,

e-mail: mlaura@fcaglp.unlp.edu.ar

* Fellow of the CONICET, Argentina.

$\star \star$ Visiting Astronomer, Complejo Astronómico El Leoncito operated under agreement between the Consejo Nacional de Investigaciones Científicas y Técnicas de la República Argentina and the National Universities of La Plata, Córdoba and San Juan.

$\star \star \star$ Member of the Carrera del Investigador Científico, CONICET, Argentina.
They also inferred that the binary system consists of a B5-7 primary and a low mass (about $0.5 M_{\odot}$ ) secondary separated by some $250 R_{\odot}$. Correlations between the slope of the Balmer progression and some asymmetries of the Balmer lines with the orbital period were also found by Iliev (1994).

As regards photometric variations, the first study was carried out by Lynds (1960), who found rapid light variations of more than $0.1^{\mathrm{m}}$ with a period of 0.85 days. Small photometric variations on several time scales were reported by Percy et al. (1988) while Pavlovski et al. (1997) found significant light and colour variations. Mennickent et al. (1994) have indicated a quasi-period of about 7 years and an amplitude up to $0.25^{\mathrm{m}}$ in the $U$ photometric band and short-term variations up to $0.1^{\mathrm{m}}$ within a few days. Finally, V923 Aql was identified as an IR variable by Dougherty \& Taylor (1994).

As many of the stars of its type, V923 Aquilae exhibits variable phenomena, most of which have not yet been satisfactorily explained. Short-term light variations could be due to nonradial pulsation (Baade 1987; Percy 1987), although other authors have attributed them to the passage of disturbances across the observable hemisphere of the star by the action of stellar rotation (Lynds 1960).

Long-term photometric and spectroscopic variations were proposed to be caused by an elongated envelope formed by the process of mass transfer from the secondary to the primary, and revolving due to the attraction of the secondary, as predicted by the binary model of Kříž \& Harmanec (1975) (Koubský et al. 1989). However, this model was later discarded by Mennickent et al. (1997), who interpreted $V / R$-photometric variations in 
terms of global density oscillations. The global density oscillation theory implies a quasi periodic $V / R$ behavior, but some observations indicate that $V / R$ variations can change period, appear or disappear entirely. Based on these facts, Gayley et al. (2001) analyzed the impact of optically thick line forces in Keplerian disks and global disk oscillations. Their calculations suggest a net retrograde precession in slightly eccentric orbits contrary to the prograde precession of non-linearly developed one-arm modes. These results reopen the discussion on the origin of $V / R$ variations.

From simultaneous visual ground-based and UV observations, Ringuelet et al. (1984) proposed a model for the extended envelope of V923 Aql with a high temperature shell located close to the star (a transition region), where highly ionized species originate, and an external cool envelope where the UV Fe II, Ca II and Na I lines are formed. But this model does not account for the observed variabilities.

To explain this scenario, and to give a better description of the structure of V923 Aql's extended envelope (Arias et al. 2000 , preliminary results) and the phenomena that are actually taking place there, we carried out a spectroscopic study of the shell line profiles, considering a semi-empirical model of the envelope (Cidale \& Ringuelet 1989) that allows us to derive some properties of the line forming regions (e.g. location of the line forming region, electron temperatures, atom columns). The same kind of solutions have proved to be suitable for the interpretation of the long-term spectrophotometric behavior of some Be stars; Moujtahid et al. (1998, 1999), found that variations in the $V$ magnitude and in the second component of the Balmer discontinuity $(\Delta \mathrm{D})$ are consistent with changes in density, extent and temperature of the circumstellar envelopes. Our main goal is to describe the regions of formation of Fe II lines in the extended envelope of V923 Aql as well as to search for any correlation of the estimated parameters with the orbital period. Moreover, we intend to propose a model for the extended atmosphere able to account for the observed variabilities.

All the observations used for the present research are presented in Sect. 2. By means of a simple ellipsoidal-layer scheme for the extended envelope, described in Sect. 3, we derive average distances from the central star to regions of line formation as well as their physical conditions: electron temperature, atom columns and densities, the results of which are presented in Sect. 4. In Sects. 5 and 6 we discuss the time dependence of the results. We conclude that the variability observed in the Fe II lines of V923 Aql may be interpreted in terms of a stellar wind asymmetrically distorted by the secondary's gravitational field. Variations of Balmer progression and some of the asymmetries of Balmer lines are attributed to the revolution of an elongated envelope while the $V / R$ variabilities are related to the subsonic wind structure.

\section{Observations}

Ultraviolet and photographic spectra taken from 1980 to 1999 were analysed. Tables 1 and 2 list information on date of observation, resolution, spectral range, etc., for UV and visual ranges respectively.
Table 1. IUE observations of V923 Aql.

\begin{tabular}{lcc}
\hline \hline Image & Date & $\begin{array}{c}\text { JD } \\
-2440000\end{array}$ \\
& & 4801.030 \\
\hline LWR11064 & $15 / 07 / 81$ & 5183.008 \\
LWR13827 & $01 / 08 / 82$ & 7829.096 \\
LWP16693 & $29 / 10 / 89$ & 8012.708 \\
LWP17843 & $01 / 05 / 90$ & 8026.509 \\
LWP17905 & $15 / 05 / 90$ & $806 / 977$ \\
LWP23361 & $23 / 06 / 92$ & 8796.677 \\
\hline SWP14478 & $15 / 07 / 81$ & 4801.041 \\
SWP17550 & $01 / 08 / 82$ & 5182.997 \\
SWP21152 & $24 / 09 / 83$ & 5602.158 \\
SWP37481 & $29 / 10 / 89$ & 7829.082 \\
SWP37482 & $29 / 10 / 89$ & 7829.121 \\
SWP37484 & $29 / 10 / 89$ & 7829.202 \\
SWP38710 & $01 / 05 / 90$ & 8012.691 \\
SWP38712 & $01 / 05 / 90$ & 8012.726 \\
SWP44983 & $23 / 06 / 92$ & 8796.648 \\
\hline
\end{tabular}

Note: Wavelength range for SWP images: $1150 \AA-1980 \AA$, and for LWP and LWR images: $1850 \AA-3350 \AA$, Res. $0.2 \AA$.

The selected material consists of fifteen high dispersion UV images taken from the INES (International ultaviolet explorer Newly Extrated Spectra) database, three spectrophotographic plates taken simultaneously in the blue and red ranges at CTIO (Cerro Tololo Interamerican Observatory), Chile on July, 1980 and 1981 and a series of $\mathrm{H} \alpha$ line observations obtained by Ringuelet, with the coudé spectrograph attached to the 1.52-m telescope at ESO (European Southern Observatory), Chile on August 1982, by Sahade \& Barbá, with the $1.5-\mathrm{m}$ telescope at CTIO on June and July 1991 and by us with the REOSC échelle spectrograph at the 2.15-m telescope at CASLEO (Complejo Astronómico El Leoncito, Argentina) in 1995 and 1999. Plates D1235 are simultaneous with the IUE images: LWR11064 and SWP14478 and plate F7998 is simultaneous with LWR13827 and SWP17550. The UV line spectrum of the star is essentially one of absorption features. It displays a rich spectrum in Fe II lines with Doppler profiles showing $R V$ and intensity variations (Fig. 1). The $\mathrm{H} \alpha$ profile displays prominent emissions and a very deep narrow central absorption. The line shows long-term $V / R$ variations, which are evident through all the observations shown in Fig. 2. The visual line spectrum was described in detail by Ringuelet \& Sahade (1981).

\section{Methodology}

The shape of the $\mathrm{H} \alpha$ line profile in the spectra of V923 Aql, a double peaked emission with a deep central absorption component, is consistent with a slow wind with low velocity gradients in the regions close to the photosphere (Cidale \& Ringuelet 1993). This result combined with the analysis of Fe II and $\mathrm{Mg}$ II sharp resonance lines (Cidale 1998) allows us to state that there is no evidence of strong outflow phenomena in the atmosphere of V923 Aql. Therefore, the extended envelope of the star could be described, in a first approximation, by a static ellipsoidal slab-like model (Cidale \& Ringuelet 1989), where the 


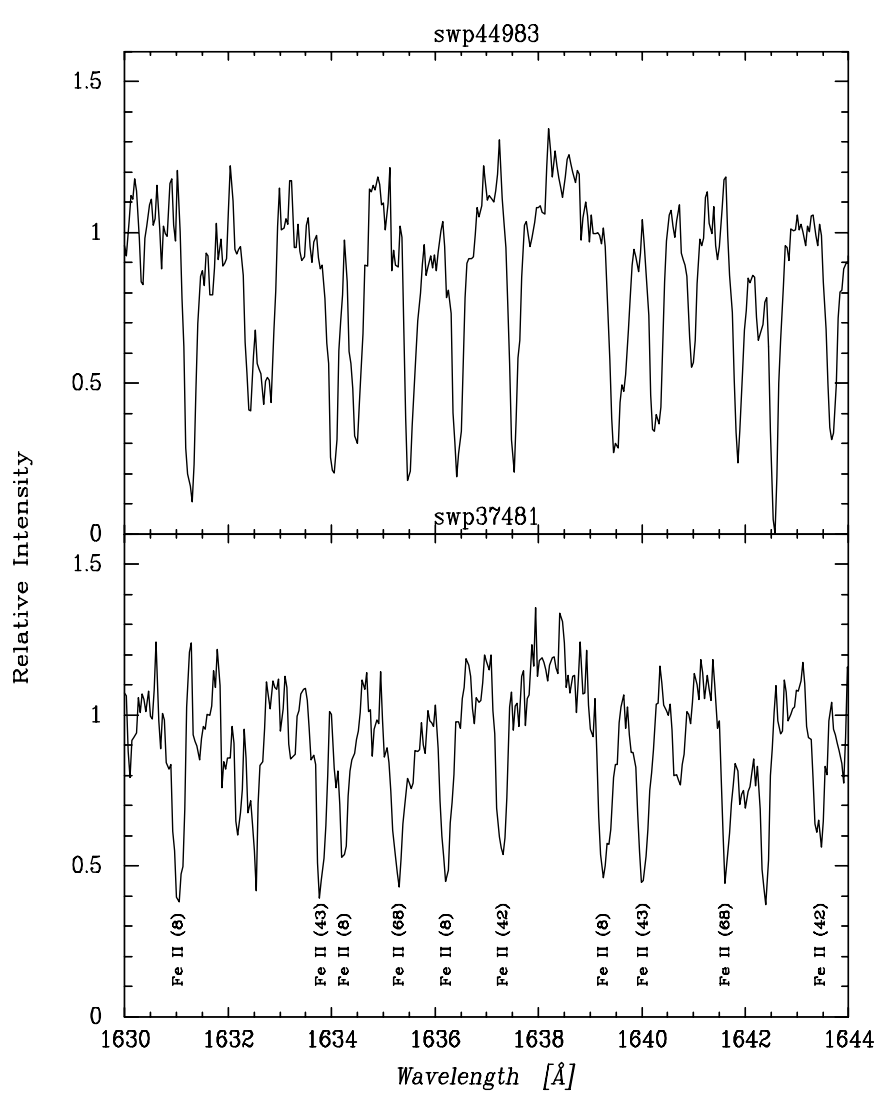

Fig. 1. Fe II line profiles for two different epochs.

Table 2. Visual observations of V923 Aql.

\begin{tabular}{|c|c|c|c|c|c|}
\hline $\begin{array}{l}\text { Plate or } \\
\text { Image }\end{array}$ & Source & Date & $\begin{array}{r}\text { JD } \\
-2440000\end{array}$ & $\begin{array}{l}\text { Disp. } \\
{[\AA / \mathrm{mm}]}\end{array}$ & $\begin{array}{l}\text { Spectral } \\
\text { range }[\AA]\end{array}$ \\
\hline \multirow[t]{2}{*}{ D1172 } & CTIO & $30 / 07 / 80$ & 4450.748 & 9.0 & $3400-4900$ \\
\hline & CTIO & $30 / 07 / 80$ & 4450.740 & 18.0 & $5700-6600$ \\
\hline \multirow[t]{2}{*}{ D1235 } & CTIO & $15 / 07 / 81$ & 4801.729 & 9.6 & $3440-4920$ \\
\hline & CTIO & $15 / 07 / 81$ & 4801.720 & 19.2 & $5700-6600$ \\
\hline \multirow[t]{2}{*}{ D1242 } & CTIO & $18 / 07 / 81$ & 4804.708 & 9.6 & $3440-4920$ \\
\hline & CTIO & $18 / 07 / 81$ & 4804.708 & 19.2 & $5700-6600$ \\
\hline F7998 & ESO & $01 / 08 / 82$ & 5183.015 & 20.0 & $5700-6600$ \\
\hline 290691a & CTIO & 29/06/91 & 8436.884 & 2.7 & $5700-6600$ \\
\hline $290691 \mathrm{~b}$ & CTIO & 29/06/91 & 8436.913 & 2.7 & $5700-6600$ \\
\hline 020791 & CTIO & $02 / 07 / 91$ & 8439.858 & 2.7 & $5700-6600$ \\
\hline 100495 & CASLEO & $10 / 04 / 95$ & 9817.913 & 0.2 & $4900-6900$ \\
\hline 290999 & CASLEO & 29/09/99 & 11451.000 & 0.2 & $4900-6900$ \\
\hline
\end{tabular}

line radiation flux that emerges from the star plus the envelope is given by the expression:

$$
\begin{aligned}
& F_{l} / F^{*}=\mathrm{e}^{-\tau}+\alpha\left[1-2 E_{3}(2 \tau)\right] \text { for } \tau<1, \\
& F_{l} / F^{*}=\mathrm{e}^{-\tau}-2 \alpha H(\tau) \text { for } \tau>1
\end{aligned}
$$

where

$\alpha=\left(\frac{R_{e}}{R_{*}}\right)^{2} \frac{S_{l} \phi}{I_{v}^{*}}$,

depends on the geometry of the envelope.
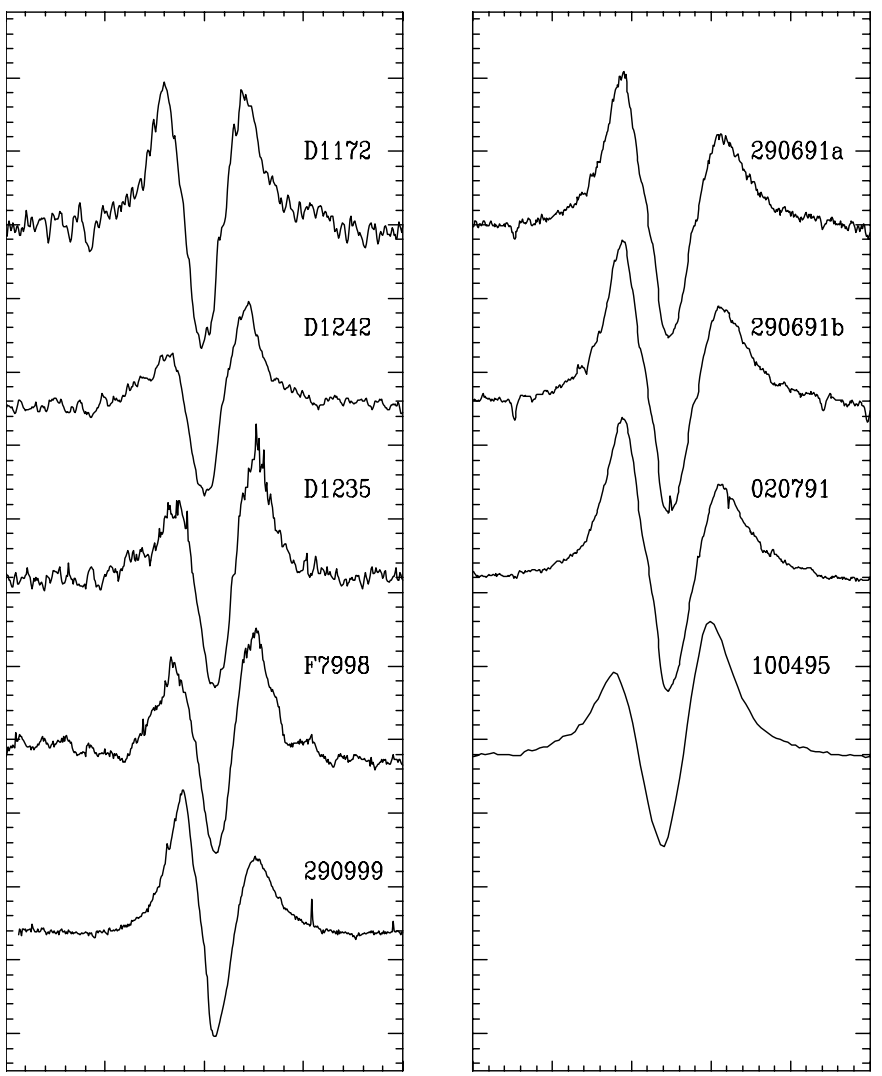

Fig. 2. $\mathrm{H} \alpha$ line profiles of V923 Aql.

Expressions (1) and (2) represent the total line flux, $F_{l}$, observed at a distance $D$ from the star in terms of the line optical depth, $\tau$, the line source function, $S_{l}$, and the geometrical factor, $\left(\frac{R_{e}}{R_{*}}\right)^{2} \phi$, for a transparent and a thick gaseous media, respectively. $R_{e}$ is the mean distance of the line-forming region to the central star, $R_{*}$ is the stellar radius, $\phi$ gives a relation between the polar and equatorial radius of the extended envelope, $F^{*}$ and $I_{v}^{*}$ are the continuous monochromatic flux and intensity corresponding to the photospheric layers.

The parameter $\alpha$ can be determined from observations and permits us to derive physical conditions, such as, line optical depth, atom columns, electron temperature and the ratio $\frac{R_{e}}{R_{*}}$.

The line source function is evaluated at $\tau=0$ and may be expressed in terms of the processes that dominate the line formation (cf. Mihalas 1978). In a two level atom model controlled by radiative processes $(\mathrm{R}-\mathrm{C})$, we have

$S_{l}(0)=\left(\frac{\eta}{1+\eta}\right)^{1 / 2} B^{*}$,

and, for collisionally controlled lines (C-C),

(2) $S_{L}(0)=\left(\frac{\epsilon}{1+\epsilon}\right)^{1 / 2} B_{v}\left(T_{\mathrm{e}}\right)$.

Expressions for $\epsilon$ and $\eta$, the relative rates for direct collisional de-excitation and photoelectric ionization followed by recombination respectively, $B_{v}$, the Planck function, and $B^{*}$, are taken from Mihalas (1978). The C-C case would provide information about the electron temperature of the line formation region, $T_{\mathrm{e}}$. 


\section{Results}

\subsection{Parameters of the circumstellar envelope: $\alpha$ and $\tau$}

By measuring the central line absorption depth, $r=\frac{F^{*}-F_{l}}{F^{*}}$, of pairs of lines belonging to the same multiplet and having their optical depth ratio, $\frac{\tau_{1}}{\tau_{2}}=\frac{\lambda_{1} g f_{1}}{\lambda_{2} g f_{2}}$, we calculated the " $\alpha$ " parameter that better fits expressions (1) and (2). The wavelengths, $\lambda$, and the oscillator strengths, $g f$, are known values (Kurucz 1981; Wiese 1985; Wiese \& Martin 1980). In general, more than two lines of the same multiplet are available, hence, accurate values of $\alpha$ and $\tau$ can be obtained (a detailed description of the method used is presented in Cidale \& Ringuelet 1989). The corresponding values of $\alpha$ and $\tau$ for Fe II lines are presented in Tables 4-6. The different columns give, for every measured line on each plate or image, the laboratory wavelength, the multiplet number, the central absorption depth, the $\alpha$ parameter and the line optical depth. When the quality of the spectrum was poor or the feature was blended it was not possible to obtain any value.

\section{2. $N_{i}, T_{\mathrm{e}}$ and $\frac{R_{e}}{R_{*}}$}

Once we know the values of the optical depth $\tau$ in the center of the line, we are able to calculate atom columns of the lower level. Integrating the formal expression of the line optical depth along the whole atmosphere, given by:

$\tau=\int n_{\mathrm{D}} n_{i} \mathrm{~d} s$

with $n_{\mathrm{D}}=\frac{\sqrt{\pi} e^{2} f H(a, v)}{m c \Delta v_{\mathrm{D}}}$, the line absorption coefficient, $n_{i}$ the number of absorbing atoms per cubic centimeter, $H(a, v)$ the Hjerting function and $\Delta v_{\mathrm{D}}$ the Doppler width, we obtain,

$\tau=\frac{1.5 \times 10^{-15} g f \lambda}{V_{\mathrm{D}}} \frac{N_{i}}{g_{i}}$

where $N_{i}$ is the atom column in $\mathrm{cm}^{-2}, \lambda$ is in $\AA$ and $V_{\mathrm{D}}$ is the Doppler velocity in $\mathrm{km} \mathrm{s}^{-1} . V_{\mathrm{D}}$ is determined through the Goldberg's method by measuring the line Doppler widths (cf. Jefferies 1968).

Knowing $\tau$ and $V_{\mathrm{D}}$ we calculate $\left(N_{i} / g_{i}\right)$ through expression (6).

Fundamental parameters of V923 Aql have been derived using the BCD spectrophotometric system (Chalonge \& Divan 1952) by Moujtahid et al. (1998), who found that the photospheric layers of the star are characterized by $T_{\text {eff }}=13770 \mathrm{~K}$ and $\log g=4.03$. This temperature is somewhat lower than the one deduced by Ringuelet \& Sahade (1981): $T_{\text {eff }}=15000 \mathrm{~K}$ and $\log g=4.0$. Based on these results, we adopt a Kurucz's (1979) model with $T_{\text {eff }}=14000 \mathrm{~K}$, to represent the continuum flux of the star, and calculate photoelectric and collisional rates for Fe II lines.

We conclude that multiplets UV(1), UV(62), UV(63) and $\mathrm{UV}(64)$ would be collisionally dominated for electron densities greater than $3 \times 10^{11} \mathrm{~cm}^{-3}$, while the rest of the lines, originating in the UV and visual regions are photoelectrically controlled for any possible value of $N_{\mathrm{e}}$ in the extended envelope $\left(N_{\mathrm{e}}<10^{13} \mathrm{~cm}^{-3}\right.$, Moujtahid et al. 1999).
Since the electron temperature is related to collisional processes and, therefore, to local conditions, only in the case of a line source function collisionally controlled is the population of the levels determined by the Boltzmann distribution and it is possible to derive $T_{\mathrm{e}}$. When we plot $\log \left(N_{i} / g_{i}\right)$ against the excitation potential of the lower level, $\chi_{i}, T_{\mathrm{e}}$ is determined through the slope of the straight line which is a mean square solution for the plotted relation. From the IUE images (LWP and LWR), we obtained a mean value for the electron temperature of about $10000 \mathrm{~K}$.

The mean distances $\left(R_{e} / R_{*}\right)$, derived from the parameter $\alpha$ according to expression (3), are presented in Tables 7-9. These tables list the plate or image number, the date of observation, the estimated values of the distance to the line-forming region averaged for multiplets with similar excitation energy and the orbital phase calculated according to the ephemeris given by Koubský et al. (1989): $T_{\max }(R V)=\mathrm{JD} 2424805+214.756 d E$, where the zero phase corresponds to the maximum radial velocity. For a qualitative discussion, we just listed the lowest limits of $R_{e} / R_{*}$, which correspond to the factor $\phi=1$.

To estimate the error in the measurements for the UV lines, the line profile stability of the high resolution IUE images has been assessed. This was possible using SWP images taken in rapid succession (i.e. SWP37481, SWP37482 and SWP37485 on Oct. 9, 1980 and SWP38710 and SWP38712 on May 1, 1981). For most lines, the differences found in the central absorption depths from image to image were of the order of $10 \%$. An uncertainty of $10 \%$ on the central line absorption depth introduces an error of $20 \%$ on the estimated ratio $\left(R_{e} / R_{*}\right)$ (Cruzado 1994).

\section{3. $H \alpha$ V/R variations}

We determined variations in the intensity of the violet $(V / C)$ and the red $(R / C)$ emission relative to the local continuum, and the corresponding $V / R$ ratio $\left(V / R=I_{v} / I_{r}\right)$. To obtain a better estimate of the long-term periodic variation, these data were combined with the few records on $V / R$ ratio found in the literature. All the values are presented in Table 3 . This table lists Julian date of the observation, $V / C$ and $R / C$ ratios, when available and the $V / R$ ratio. References to the source where data was taken are shown in the last column.

We determined a period of 6.8 years for the $V / R$ variations using the Lomb-Scargle periodogram (Horne \& Baliunas 1986). This period is in agreement with the cycle of 6.5 years estimated by Merrill \& Lowen (1953) for radial velocities but larger than the long-term cycle in the radial velocity, with a quasiperiod of 5.8 years, suggested by Koubský et al. (1989). Figure 3 depicts the corresponding long-term $V / R$ variations.

\section{Discussion}

\subsection{The structure of the envelope}

From the computed ratio $\left(R_{e} / R_{*}\right)$, for the photographic range, we can distinguish two line formation regions for Fe II lines, which are located around 1.5 and 3.5 stellar radii, approximately (Table 7). These values correspond to different 
Table 3. $V / R$ variation of the $\mathrm{H} \alpha$ profile.

\begin{tabular}{rcccc}
\hline \hline JD-2440000 & $V / C$ & $R / C$ & $V / R$ & Reference \\
\hline 3009.500 & - & - & $0.88^{*}$ & Fontaine et al. (1982) \\
3007.500 & - & - & $0.88^{*}$ & Fontaine et al. (1982) \\
3006.500 & - & - & $0.89^{*}$ & Fontaine et al. (1982) \\
4450.748 & 1.84 & 1.77 & 1.04 & this work \\
4801.729 & 1.32 & 1.73 & 0.76 & this work \\
4804.708 & 1.26 & 1.63 & 0.77 & this work \\
5183.015 & 1.59 & 1.78 & 0.89 & this work \\
5610.698 & 1.62 & 1.46 & 1.11 & Ballereau et al. (1987) \\
5611.710 & 1.60 & 1.50 & 1.07 & Ballereau et al. (1987) \\
5619.678 & 1.65 & 1.40 & 1.18 & Ballereau et al. (1987) \\
6640.486 & 1.80 & 1.60 & 1.13 & Denizman et al. (1994) \\
6930.580 & 2.20 & 1.90 & 1.16 & Denizman et al. (1994) \\
6990.541 & 1.70 & 1.70 & 1.00 & Denizman et al. (1994) \\
7490.000 & 1.48 & 2.56 & $0.58^{*}$ & Doazan et al. (1991) \\
8436.884 & 2.07 & 1.59 & 1.30 & this work \\
8436.913 & 2.02 & 1.64 & 1.25 & this work \\
8439.858 & 2.05 & 1.61 & 1.29 & this work \\
9095.500 & 2.11 & 1.77 & $1.20^{*}$ & Hanuschik et al. (1996) \\
9239.500 & 2.15 & 1.65 & $1.30^{*}$ & Hanuschik et al. (1996) \\
9817.913 & 1.87 & 2.38 & 0.79 & this work \\
11451.00 & 2.01 & 1.53 & 1.32 & this work \\
\hline
\end{tabular}

Note: The symbol $(*)$ indicates intensity measurements taken by us on published spectra.

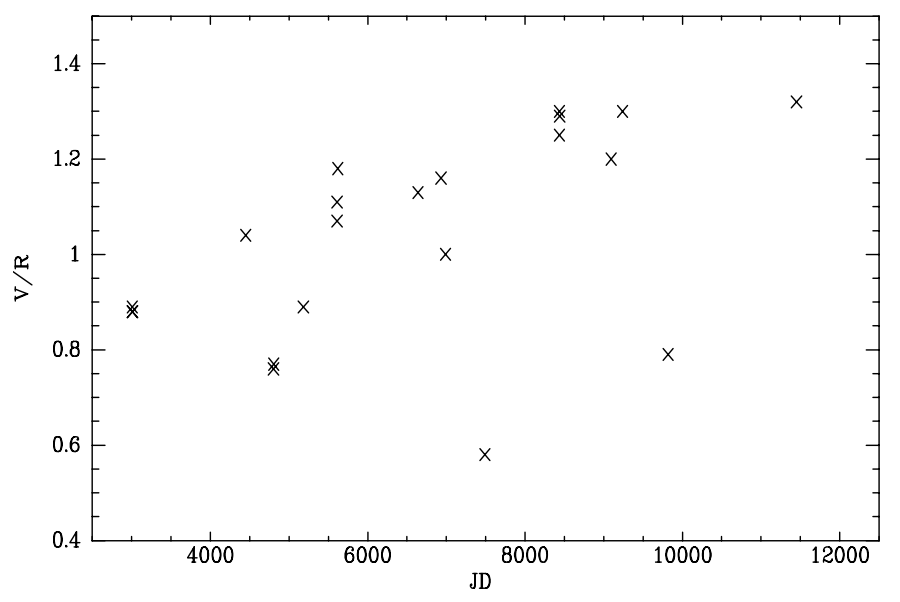

Fig. 3. Long-term $V / R$ variations observed in the $\mathrm{H} \alpha$ profile.

groups of lines which are classified according to their excitation energy. In our model, multiplets (114) and (144) with higher excitation energy $\left(E_{l} \approx 4.4 \mathrm{eV}\right)$ are formed in a region closer to the central star than those with lower excitation energy (multiplets (27), (28), (37) and (38) with $E_{l} \approx 2.7 \mathrm{eV}$ ).

The mean extent of the line-forming region for LWR and LWP spectra is around 3.25 stellar radii, and its value varies with time from 2 to 4.5 stellar radii (Table 8 ). The UV line optical depths are all greater than 1 and the obtained values for $\log \left(N_{i} / g_{i}\right)$ are similar for all the images, averaging $13 \mathrm{dex}$.

The $\alpha$ values obtained for the UV LWP-LWR multiplets are lower than the ones calculated for the photographic region; the line profiles are deeper and the optical depths are much larger than the photographic ones (Table 5).

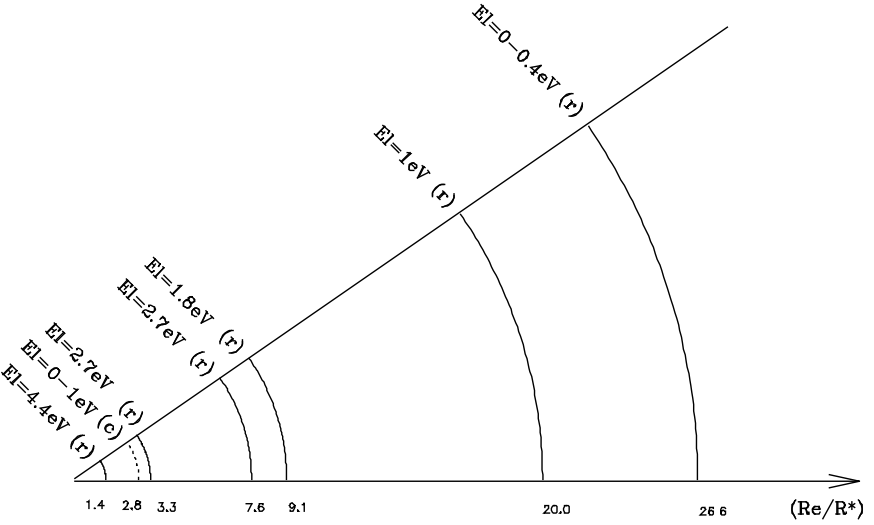

Fig. 4. Relative position of the regions of formation of Fe II lines in the outer envelope of V923 Aquilae. $E_{l}$ is the excitation energy of the lower level and (r) and (c) indicate multiplets R-C or C-C, respectively.

The line optical depths in SWP images are also larger than 1 , indicating in some cases saturation effects. $\left(R_{e} / R_{*}\right)$ ratios estimated for R-C lines in SWP spectra (Table 9) are about seven times higher than those measured for the $\mathrm{C}$-C lines in LWP and LWR images. This difference is considerably larger than the variations of the values of $\left(R_{e} / R_{*}\right)$, indicating a different region of formation for these lines. Hence, for multiplets with similar lower excitation energies $(\approx 1 \mathrm{eV})$ : the collisional lines form closer to the star than radiative ones.

A scheme of the different formation regions in the envelope of V923 Aql. is shown in Fig. 4. Our results would indicate that the Fe II forming region extends from 1.5 to 35 stellar radii (both values are lower limits).

\subsection{Time variations}

Assuming that the orbital period of V923 Aql binary system is 214.756 days (Koubský et al. 1989), we test the possibility that some of the observed time variabilities correlate with this orbital period.

It can be noticed that the calculated mean distance from the central star to Fe II formation regions varies with time (Tables 7-9). Variations of around $100 \%$ and larger than $50 \%$ are found for the ratio $\left(R_{e} / R_{*}\right)$ in the inner and outer regions, respectively. The observed variation is much larger than the estimated error of $\sim 20 \%$ for $\Delta\left(R_{e} / R_{*}\right)$. This behavior is presented in Figs. 5 and 6.

Figure 5 illustrates the variations of $\left(R_{e} / R_{*}\right)$ against the orbital phase corresponding to the visual and LWP-LWR IUE data presented in Tables 7 and 8, together with the orbital velocity curve given by Koubský et al. (1989) on top. Apparently, there is a dependence of the ratio $\left(R_{e} / R_{*}\right)$ with the phase; this ratio increases between phases 0.0 and 0.2 and between phases 0.6 and 1.0 .

Temporal variations of $\left(R_{e} / R_{*}\right)$ ratios for SWP images have a similar trend as for LWP-LWR images, but with larger amplitudes (Table 9). The amplitude of the variation depends on the location of the line forming region. The outer the region, the larger the amplitude. 
Table 4. Values of $r, \alpha$ and $\tau$, corresponding to the photographic region.

\begin{tabular}{|c|c|c|c|c|c|c|c|c|c|c|}
\hline \multirow[b]{2}{*}{$\lambda[\AA]$} & \multirow[b]{2}{*}{ Multiplet } & \multicolumn{3}{|c|}{ 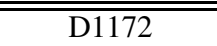 } & \multicolumn{3}{|c|}{$\overline{\bar{D} 1235}$} & \multicolumn{3}{|c|}{$\overline{\text { D1242 }}$} \\
\hline & & $r$ & $\alpha$ & $\tau$ & $r$ & $\alpha$ & $\tau$ & $r$ & $\alpha$ & $\tau$ \\
\hline 4233.172 & (27) & 0.69 & 0.30 & 5.20 & 0.31 & 0.32 & 0.94 & 0.58 & 0.48 & 4.50 \\
\hline 4351.768 & & 0.70 & 0.30 & 4.25 & 0.26 & 0.32 & 0.80 & 0.49 & 0.48 & 3.69 \\
\hline 4416.830 & & 0.44 & 0.30 & 1.30 & & - & & 0.25 & 0.48 & 1.23 \\
\hline 4303.176 & & 0.56 & 0.30 & 1.85 & & - & & 0.30 & 0.48 & 1.43 \\
\hline 4385.387 & & 0.50 & 0.30 & 1.56 & & - & & 0.34 & 0.48 & 1.60 \\
\hline 4178.862 & (28) & 0.45 & 0.55 & 5.45 & 0.18 & 0.84 & 7.25 & & - & \\
\hline 4296.572 & & 0.28 & 0.55 & 1.65 & 0.10 & 0.84 & 2.20 & & - & \\
\hline 4122.668 & & 0.15 & 0.55 & 1.12 & & - & & & - & \\
\hline 4629.339 & (37) & 0.50 & 0.42 & 2.28 & 0.21 & 0.32 & 0.68 & 0.34 & 0.32 & 1.04 \\
\hline 4555.893 & & 0.56 & 0.42 & 2.97 & 0.17 & 0.32 & 0.58 & 0.27 & 0.32 & 0.83 \\
\hline 4515.339 & & 0.35 & 0.42 & 1.42 & 0.18 & 0.32 & 0.60 & 0.37 & 0.32 & 1.13 \\
\hline 4491.405 & & 0.30 & 0.42 & 1.23 & & - & & 0.19 & 0.32 & 0.63 \\
\hline 4520.224 & & 0.38 & 0.42 & 1.55 & & - & & 0.28 & 0.32 & 0.86 \\
\hline 4583.837 & (38) & 0.69 & 0.28 & 2.90 & 0.33 & 0.30 & 0.94 & 0.53 & 0.44 & 2.57 \\
\hline 4549.474 & & 0.72 & 0.28 & 4.15 & & - & & 0.57 & 0.44 & 4.30 \\
\hline 4522.634 & & 0.66 & 0.28 & 2.55 & 0.26 & 0.30 & 0.75 & 0.45 & 0.44 & 2.00 \\
\hline 4508.288 & & 0.49 & 0.28 & 1.43 & 0.20 & 0.30 & 0.60 & 0.35 & 0.44 & 1.47 \\
\hline 3493.470 & (114) & 0.61 & 0.36 & 2.73 & 0.43 & 0.44 & 1.60 & 0.45 & 0.55 & 4.20 \\
\hline 3468.677 & & 0.40 & 0.36 & 1.27 & 0.23 & 0.44 & 0.75 & 0.33 & 0.55 & 1.92 \\
\hline 3621.270 & (144) & 0.42 & 0.49 & 1.70 & 0.28 & 0.32 & 0.85 & 0.22 & 0.55 & 1.40 \\
\hline 3624.893 & & 0.32 & 0.49 & 1.14 & 0.17 & 0.32 & 0.56 & 0.18 & 0.55 & 1.23 \\
\hline
\end{tabular}

Table 5. Values for $r, \alpha$ and $\tau$ corresponding to LWR and LWR images.

\begin{tabular}{|c|c|c|c|c|c|c|c|c|c|c|c|c|c|c|c|c|c|c|c|}
\hline \multirow[b]{2}{*}{$\lambda[\AA]$} & \multirow[b]{2}{*}{ Multiplet } & \multicolumn{3}{|c|}{ "LWR11064 } & \multicolumn{3}{|c|}{ LWR13827 } & \multicolumn{3}{|c|}{ LWW16693 } & \multicolumn{3}{|c|}{ "LWP17843 } & \multicolumn{3}{|c|}{ LWWP17905 } & \multicolumn{3}{|c|}{ LWP23361 } \\
\hline & & $r$ & $\alpha$ & $\tau$ & $r$ & $\alpha$ & $\tau$ & $r$ & $\alpha$ & $\tau$ & $r$ & $\alpha$ & $\tau$ & $r$ & $\alpha$ & $\tau$ & $r$ & $\alpha$ & $\tau$ \\
\hline 2585.880 & UV(1) & & - & & & - & & 0.92 & 0.05 & 3.70 & 0.96 & 0.02 & 4.40 & & - & & & - & \\
\hline 2598.369 & & 0.96 & 0.04 & 5.90 & & - & & 0.90 & 0.05 & 4.60 & & - & & 0.97 & 0.02 & 7.73 & 0.98 & 0.02 & 7.80 \\
\hline 2599.395 & & & - & & 0.97 & 0.04 & 14.50 & 0.99 & 0.05 & 13.20 & 0.98 & 0.02 & 15.50 & & - & & & - & \\
\hline 2607.086 & & 0.95 & 0.04 & 3.80 & 0.94 & 0.04 & 4.40 & 0.91 & 0.05 & 3.82 & 0.97 & 0.02 & 5.05 & & - & & 0.96 & 0.02 & 6.50 \\
\hline 2611.873 & & & - & & 0.96 & 0.04 & 5.80 & & - & & & - & & 0.98 & 0.02 & 8.72 & & - & \\
\hline 2613.820 & & & - & & 0.90 & 0.04 & 2.67 & 0.75 & 0.05 & 2.50 & & - & & 0.96 & 0.02 & 4.00 & 0.93 & 0.02 & 2.94 \\
\hline 2617.618 & & & - & & 0.79 & 0.04 & 1.76 & & - & & 0.91 & 0.02 & 2.61 & & - & & 0.94 & 0.02 & 3.07 \\
\hline 2628.291 & & 0.90 & 0.04 & 2.61 & 0.88 & 0.04 & 2.51 & 0.77 & 0.05 & 2.12 & 0.92 & 0.02 & 2.81 & & - & & 0.95 & 0.02 & 3.42 \\
\hline 2631.045 & & & - & & & - & & 0.89 & 0.05 & 2.73 & & - & & & - & & & - & \\
\hline 2631.321 & & & - & & & - & & 0.91 & 0.05 & 3.06 & 0.97 & 0.02 & 4.45 & & - & & & - & \\
\hline 2743.196 & UV(62) & 0.92 & 0.04 & 3.07 & 0.85 & 0.03 & 2.07 & 0.80 & 0.10 & 2.20 & 0.89 & 0.08 & 3.15 & 0.94 & 0.05 & 4.00 & 0.94 & 0.01 & 2.98 \\
\hline 2746.487 & & 0.97 & 0.04 & 4.95 & 0.89 & 0.03 & 2.46 & 0.87 & 0.10 & 3.20 & 0.92 & 0.08 & 5.05 & 0.95 & 0.05 & 6.49 & & - & \\
\hline 2749.324 & & 0.98 & 0.04 & 7.13 & 0.97 & 0.03 & 4.25 & 0.95 & 0.10 & 5.12 & 0.99 & 0.08 & 7.50 & & - & & 0.98 & 0.01 & 6.00 \\
\hline 2714.414 & UV(63) & 0.91 & 0.03 & 2.83 & 0.78 & 0.06 & 1.80 & & - & & 0.89 & 0.05 & 2.65 & & - & & 0.89 & 0.06 & 2.97 \\
\hline 2727.538 & & & - & & 0.91 & 0.06 & 3.22 & & - & & 0.90 & 0.05 & 2.79 & & - & & & - & \\
\hline 2736.970 & & & - & & 0.74 & 0.06 & 1.63 & & - & & 0.74 & 0.05 & 1.53 & & - & & 0.84 & 0.06 & 2.23 \\
\hline 2739.545 & & 0.98 & 0.03 & 13.73 & & - & & 0.91 & 0.10 & 5.20 & 0.95 & 0.05 & 12.30 & 0.98 & 0.06 & 6.04 & 0.96 & 0.06 & 14.40 \\
\hline 2746.978 & & & - & & 0.94 & 0.06 & 5.55 & 0.86 & 0.10 & 2.91 & & - & & 0.92 & 0.06 & 3.73 & 0.94 & 0.06 & 9.00 \\
\hline 2562.535 & UV(64) & 0.97 & 0.05 & 9.69 & 0.93 & 0.06 & 8.41 & 0.87 & 0.12 & 5.52 & 0.96 & 0.04 & 7.00 & & - & & 0.99 & 0.01 & 8.50 \\
\hline 2577.920 & & 0.89 & 0.05 & 2.85 & 0.85 & 0.06 & 2.39 & 0.72 & 0.12 & 1.80 & 0.80 & 0.04 & 1.83 & 0.90 & 0.04 & 2.84 & 0.89 & 0.01 & 2.34 \\
\hline 2582.582 & & & - & & & - & & & - & & 0.87 & 0.04 & 2.37 & 0.95 & 0.04 & 3.49 & 0.95 & 0.01 & 3.26 \\
\hline 2591.542 & & 0.92 & 0.05 & 3.40 & & - & & 0.74 & 0.12 & 1.94 & 0.89 & 0.04 & 2.66 & 0.95 & 0.04 & 3.49 & 0.93 & 0.01 & 2.83 \\
\hline
\end{tabular}


Table 6. Values for $r, \alpha$ and $\tau$ corresponding to the SWP images.

\begin{tabular}{|c|c|c|c|c|c|c|c|c|c|c|c|c|c|c|c|c|}
\hline \multirow[b]{2}{*}{$\lambda[\AA ̊]$} & \multirow[b]{2}{*}{ Multiplet } & \multicolumn{3}{|c|}{ SWP14478 } & \multicolumn{3}{|c|}{ SWP17550 } & \multicolumn{3}{|c|}{ SWP21152 } & \multicolumn{3}{|c|}{ SWP37481 } & \multicolumn{3}{|c|}{ "SWP44983 } \\
\hline & & $r$ & $\alpha$ & $\tau$ & $r$ & $\alpha$ & $\tau$ & $r$ & $\alpha$ & $\tau$ & $r$ & $\alpha$ & $\tau$ & $r$ & $\alpha$ & $\tau$ \\
\hline 1608.446 & $\mathrm{UV}(8)$ & 0.89 & 0.161 & 17.00 & 0.89 & 0.141 & 14.00 & 0.77 & 0.24 & 13.00 & 0.99 & 0.26 & 12.00 & 0.98 & 0.101 & 14.00 \\
\hline 1621.685 & & 0.84 & 0.16 & 8.50 & 0.89 & 0.14 & 6.97 & & - & & 0.74 & 0.26 & 6.00 & 0.90 & 0.10 & 7.00 \\
\hline 1631.124 & & & - & & 0.83 & 0.14 & 3.30 & 0.70 & 0.24 & 2.60 & 0.73 & 0.26 & 3.45 & & - & \\
\hline 1618.464 & & 0.89 & 0.16 & 5.10 & & - & & 0.74 & 0.24 & 3.12 & 0.67 & 0.26 & 2.44 & 0.81 & 0.10 & 2.27 \\
\hline 1629.155 & & 0.83 & 0.16 & 4.15 & 0.85 & 0.14 & 5.25 & 0.79 & 0.24 & 4.70 & 0.82 & 0.26 & 4.60 & 0.90 & 0.10 & 5.25 \\
\hline 1636.334 & & 0.86 & 0.16 & 5.60 & 0.82 & 0.14 & 3.00 & 0.73 & 0.24 & 3.07 & 0.67 & 0.26 & 2.45 & 0.87 & 0.10 & 3.31 \\
\hline 1634.353 & & 0.74 & 0.16 & 2.20 & 0.70 & 0.14 & 1.80 & 0.66 & 0.24 & 2.20 & 0.61 & 0.26 & 1.95 & 0.80 & 0.10 & 2.17 \\
\hline 1639.403 & & 0.82 & 0.16 & 3.40 & 0.72 & 0.14 & 1.92 & 0.66 & 0.24 & 2.17 & 0.66 & 0.26 & 2.30 & 0.81 & 0.10 & 2.33 \\
\hline 1272.638 & UV(9) & 0.80 & 0.17 & 3.19 & 0.56 & 0.27 & 1.69 & & - & & 0.73 & 0.25 & 3.14 & 0.79 & 0.11 & 2.32 \\
\hline 1267.437 & & 0.82 & 0.17 & 8.10 & 0.67 & 0.27 & 3.32 & 0.72 & 0.28 & 4.55 & & - & & & - & \\
\hline 1272.001 & & & - & & 0.59 & 0.27 & 1.87 & 0.72 & 0.28 & 4.10 & & - & & 0.89 & 0.11 & 5.60 \\
\hline 1275.801 & & 0.83 & 0.17 & 5.80 & & - & & 0.71 & 0.28 & 3.25 & 0.94 & 0.25 & 4.65 & & & \\
\hline 1275.144 & & 0.69 & 0.17 & 1.93 & & - & & & - & & 0.66 & 0.25 & 2.20 & 0.80 & 0.11 & 2.33 \\
\hline 1702.045 & UV(38) & 0.88 & 0.151 & 17.00 & 0.88 & 0.251 & 16.00 & 0.84 & 0.272 & 21.00 & 0.76 & 0.30 & 11.00 & 0.92 & 0.162 & 24.00 \\
\hline 1713.002 & & & - & & & - & & 0.83 & 0.27 & 11.00 & 0.70 & 0.30 & 6.00 & 0.91 & 0.16 & 11.00 \\
\hline 1720.621 & & 0.85 & 0.15 & 6.14 & & - & & 0.73 & 0.27 & 7.90 & 0.72 & 0.30 & 4.00 & 0.84 & 0.16 & 8.00 \\
\hline 1726.394 & & 0.85 & 0.15 & 4.46 & 0.74 & 0.25 & 3.54 & & - & & 0.69 & 0.30 & 3.40 & & - & \\
\hline 1696.800 & & 0.77 & 0.15 & 2.44 & 0.68 & 0.25 & 2.40 & 0.71 & 0.27 & 3.17 & 0.55 & 0.30 & 1.82 & 0.82 & 0.16 & 3.55 \\
\hline 1708.627 & & 0.74 & 0.15 & 2.15 & 0.70 & 0.25 & 2.72 & 0.72 & 0.27 & 3.44 & & - & & 0.81 & 0.16 & 3.17 \\
\hline 1718.123 & & 0.69 & 0.15 & 1.76 & 0.58 & 0.25 & 1.67 & 0.50 & 0.27 & 1.42 & & - & & 0.60 & 0.16 & 1.37 \\
\hline 1659.487 & UV(40) & 0.78 & 0.23 & 8.70 & 0.75 & 0.25 & 5.30 & 0.67 & 0.33 & 8.00 & & - & & & - & \\
\hline 1663.226 & & 0.79 & 0.23 & 4.39 & 0.69 & 0.25 & 2.60 & 0.67 & 0.33 & 4.50 & 0.72 & 0.27 & 3.65 & 0.83 & 0.16 & 4.54 \\
\hline 1674.716 & & & - & & & - & & 0.61 & 0.33 & 2.47 & & - & & & - & \\
\hline 1686.455 & & 0.75 & 0.23 & 3.25 & & - & & & - & & 0.68 & 0.27 & 2.63 & 0.81 & 0.16 & 3.39 \\
\hline 1658.785 & UV(41) & 0.88 & 0.27 & 6.64 & 0.78 & 0.22 & 4.70 & 0.72 & 0.33 & 4.35 & 0.69 & 0.31 & 4.45 & & - & \\
\hline 1685.953 & & 0.73 & 0.27 & 4.67 & 0.71 & 0.22 & 2.45 & 0.59 & 0.33 & 2.34 & 0.66 & 0.31 & 2.90 & & - & \\
\hline 1693.936 & & 0.63 & 0.27 & 2.17 & & - & & 0.54 & 0.33 & 1.92 & 0.55 & 0.31 & 1.86 & & - & \\
\hline 1612.814 & UV(43) & & - & & 0.78 & 0.22 & 7.00 & & - & & 0.98 & 0.29 & 7.00 & & - & \\
\hline 1623.092 & & 0.72 & 0.19 & 2.27 & 0.75 & 0.22 & 3.10 & & - & & & - & & 0.78 & 0.11 & 2.15 \\
\hline 1625.525 & & 0.83 & 0.19 & 5.97 & 0.76 & 0.22 & 3.25 & 0.74 & 0.26 & 3.90 & 0.71 & 0.29 & 4.30 & 0.89 & 0.11 & 5.60 \\
\hline 1633.907 & & 0.80 & 0.19 & 3.60 & 0.77 & 0.22 & 3.75 & 0.71 & 0.26 & 3.07 & 0.71 & 0.29 & 3.90 & 0.86 & 0.11 & 3.19 \\
\hline 1640.167 & & & - & & 0.76 & 0.22 & 3.32 & 0.68 & 0.26 & 2.51 & 0.69 & 0.29 & 3.04 & & - & \\
\hline 1569.670 & UV(44) & & - & & & - & & & - & & 0.61 & 0.34 & 2.60 & 0.87 & 0.10 & 3.40 \\
\hline 1580.635 & & & - & & & - & & 0.76 & 0.25 & 4.65 & 0.74 & 0.34 & 6.00 & & - & \\
\hline 1584.954 & & 0.79 & 0.20 & 3.75 & 0.76 & 0.21 & 3.13 & 0.72 & 0.25 & 2.92 & 0.66 & 0.34 & 4.20 & & - & \\
\hline 1588.295 & & 0.75 & 0.20 & 2.76 & 0.67 & 0.21 & 1.99 & 0.64 & 0.25 & 2.07 & 0.63 & 0.34 & 2.90 & 0.93 & 0.10 & 5.70 \\
\hline 1566.825 & & 0.83 & 0.20 & 4.85 & 0.83 & 0.21 & 4.80 & 0.72 & 0.25 & 3.02 & 0.71 & 0.34 & 4.35 & & - & \\
\hline 1563.790 & UV(45) & & - & & 0.72 & 0.282 & 22.00 & 0.73 & 0.322 & 24.00 & & - & & 0.90 & 0.122 & 21.00 \\
\hline 1570.244 & & & - & & 0.72 & 0.281 & 17.00 & 0.68 & 0.32 & 18.00 & 0.70 & 0.32 & 17.69 & 0.87 & 0.12 & 14.00 \\
\hline 1568.020 & & & - & & 0.67 & 0.28 & 2.62 & 0.66 & 0.32 & 3.17 & 0.65 & 0.32 & 2.90 & 0.80 & 0.12 & 2.42 \\
\hline 1859.744 & UV(65) & 0.76 & 0.24 & 5.99 & & - & & 0.67 & 0.34 & 6.93 & & - & & 0.74 & 0.26 & 1.57 \\
\hline 1851.517 & & 0.45 & 0.24 & 1.14 & & - & & 0.40 & 0.34 & 1.32 & & - & & 0.54 & 0.26 & 9.00 \\
\hline 1635.389 & UV(68) & & - & & 0.76 & 0.24 & 6.00 & 0.73 & 0.29 & 8.00 & & - & & & - & \\
\hline 1641.761 & & 0.81 & 0.20 & 4.85 & 0.71 & 0.24 & 2.63 & 0.71 & 0.29 & 4.15 & & - & & & - & \\
\hline 1646.187 & & 0.77 & 0.20 & 3.07 & & - & & 0.61 & 0.29 & 2.17 & 0.58 & 0.32 & 2.16 & & - & \\
\hline 1647.161 & & 0.75 & 0.20 & 2.71 & 0.57 & 0.24 & 1.59 & 0.59 & 0.29 & 2.00 & & - & & 0.85 & 0.10 & 2.90 \\
\hline 1649.583 & & 0.90 & 0.20 & 4.90 & & - & & & - & & & - & & 0.98 & 0.10 & 5.60 \\
\hline 1650.709 & & 0.71 & 0.20 & 2.27 & & - & & 0.59 & 0.29 & 2.00 & 0.56 & 0.32 & 1.95 & 0.86 & 0.10 & 2.98 \\
\hline 1709.678 & UV(84) & 0.82 & 0.20 & 4.85 & 0.91 & 0.10 & 5.75 & 0.89 & 0.10 & 4.10 & 0.86 & 0.35 & 9.00 & 0.94 & 0.06 & 5.95 \\
\hline 1707.411 & & 0.72 & 0.20 & 2.36 & 0.76 & 0.10 & 1.92 & 0.65 & 0.10 & 1.39 & 0.62 & 0.35 & 2.85 & 0.78 & 0.06 & 1.83 \\
\hline 1720.042 & & 0.75 & 0.20 & 2.66 & 0.75 & 0.10 & 1.86 & 0.58 & 0.10 & 1.12 & 0.65 & 0.35 & 3.85 & & - & \\
\hline 1689.821 & UV(85) & 0.57 & 0.34 & 2.20 & 0.45 & 0.43 & 1.93 & 0.49 & 0.41 & 2.07 & & - & & & - & \\
\hline 1690.781 & & & - & & 0.53 & 0.43 & 2.59 & 0.58 & 0.41 & 3.20 & 0.52 & 0.23 & 1.35 & 0.65 & 0.34 & 3.25 \\
\hline 1699.199 & & 0.67 & 0.34 & 4.35 & 0.58 & 0.43 & 3.90 & 0.51 & 0.41 & 2.27 & 0.42 & 0.23 & 1.02 & 0.62 & 0.34 & 2.67 \\
\hline 1693.477 & & & - & & & - & & 0.49 & 0.41 & 2.08 & & - & & & - & \\
\hline 1772.518 & UV(99) & 0.77 & 0.12 & 2.10 & 0.66 & 0.33 & 3.45 & & - & & 0.60 & 0.38 & 3.05 & 0.75 & 0.25 & 4.04 \\
\hline 1793.371 & & 0.70 & 0.12 & 1.69 & 0.63 & 0.33 & 2.74 & & - & & 0.56 & 0.38 & 2.47 & 0.73 & 0.25 & 3.26 \\
\hline 1798.163 & UV(142) & 0.68 & 0.32 & 4.20 & & - & & 0.62 & 0.29 & 2.26 & 0.56 & 0.44 & 3.47 & 0.72 & 0.21 & 2.52 \\
\hline 1809.316 & & 0.63 & 0.32 & 2.63 & & - & & 0.50 & 0.29 & 1.49 & 0.51 & 0.44 & 2.29 & 0.61 & 0.21 & 1.67 \\
\hline 1785.262 & UV(191) & 0.81 & 0.18 & 3.80 & 0.81 & 0.11 & 2.39 & & - & & 0.70 & 0.30 & 3.95 & 0.85 & 0.12 & 3.20 \\
\hline 1786.738 & & 0.78 & 0.18 & 2.80 & 0.73 & 0.11 & 1.79 & & - & & 0.69 & 0.30 & 3.45 & 0.80 & 0.12 & 2.43 \\
\hline
\end{tabular}




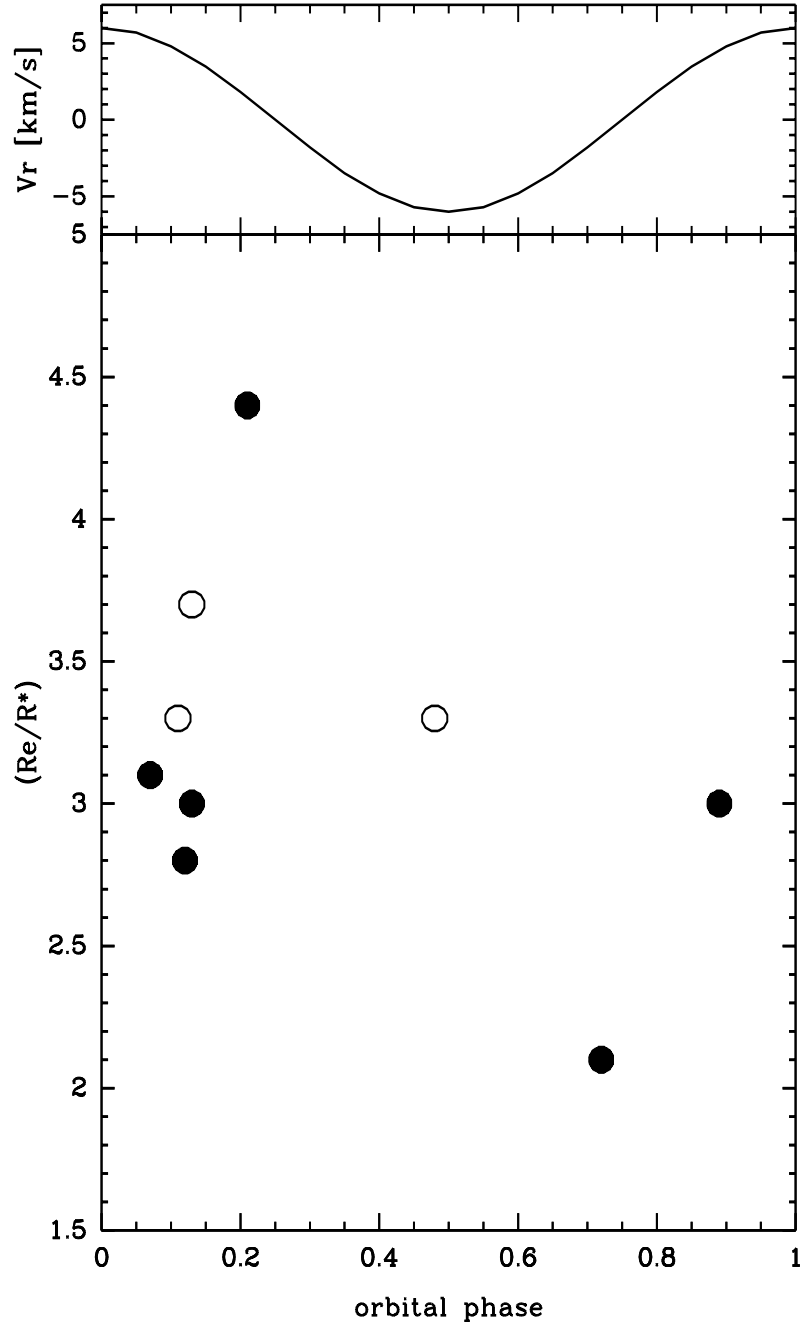

Fig. 5. Distribution of values of $\left(R_{e} / R_{*}\right)$ with orbital phase, together with radial velocity curve taken from Koubský et al. (1989) on top. (०) values of $\left(R_{e} / R_{*}\right)_{1}$ from Table $7,(\bullet)$ values of $\left(R_{e} / R_{*}\right)$ from Table 8 .

Table 7. Calculated values of $\left(R_{e} / R_{*}\right)$ for Fe II lines in the photographic region, averaged for multiplets with similar excitation energy.

\begin{tabular}{ccccc}
\hline \hline Plate & Date & $\left(R_{e} / R_{*}\right)_{1}$ & $\left(R_{e} / R_{*}\right)_{2}$ & Phase \\
\hline D1172 & $30 / 07 / 80$ & 3.3 & 1.4 & 0.48 \\
D1235 & $15 / 07 / 81$ & 3.3 & 1.4 & 0.11 \\
D1242 & $18 / 07 / 81$ & 3.7 & 1.6 & 0.13 \\
\hline
\end{tabular}

Note: $\left(R_{e} / R_{*}\right)_{1}$ is the average value of the distance to the line forming region for multiplets (27), (28), (37) y (38) $\left(E_{l} \approx 2.7 \mathrm{eV}\right)$ and $\left(R_{e} / R_{*}\right)_{2}$ is the average value for (114) and (144), $\left(E_{l} \approx 4.4 \mathrm{eV}\right)$. The last column shows the phase calculated with the period $214.756 \mathrm{~d}$ estimated by Koubský et al. (1989).

To be able to compare $\left(R_{e} / R_{*}\right)$ variations of similar amplitude, we plotted the estimated values of $\left(R_{e} / R_{*}\right)$ listed in Tables 7-9 minus the mean $\left(R_{e} / R_{*}\right)$ value for each column in Fig. 6. Figure 6a shows $\left(R_{e} / R_{*}\right)$ variation from Tables 7 and 8 and the Cols. 5 and 6 of Table 9 and Fig. $6 \mathrm{~b}$ shows the values of $\left(R_{e} / R_{*}\right)$ for the Cols. 3 and 4 of Table 9.
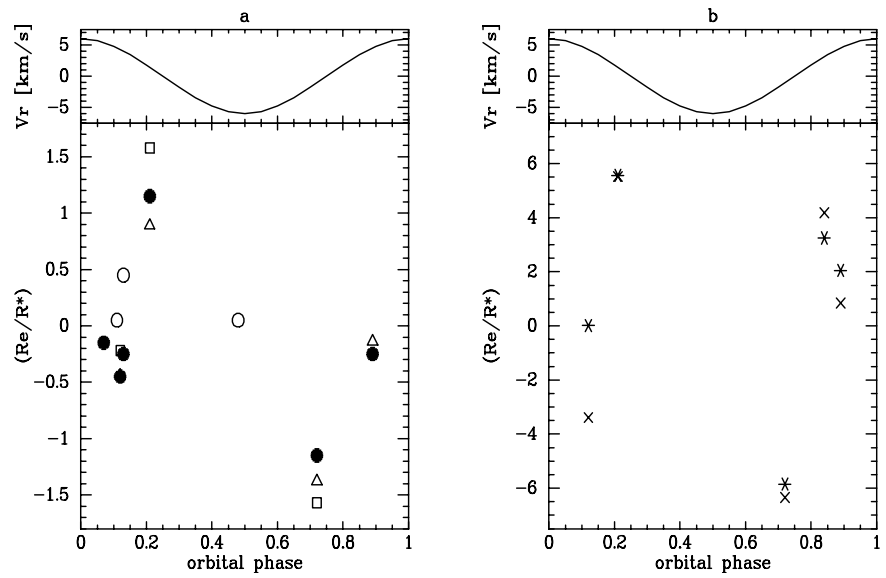

Fig. 6. Values of $\left(R_{e} / R_{*}\right)$ (from Tables 7-9) minus the corresponding average values for each column against orbital phase. a) Variations of $\left(R_{e} / R_{*}\right)$ with similar amplitude for visual $(\circ)\left(\left(R_{e} / R_{*}\right)_{1}\right.$ from Table 7), LWP-LWR $(\bullet)$ (Table 8) and SWP images $(\triangle$ and $\square$ ) $\left(\left(R_{e} / R_{*}\right)_{3}\right.$ and $\left(R_{e} / R_{*}\right)_{4}$ from Table 9$)$, b) Variations of $\left(R_{e} / R_{*}\right)$ with similar amplitude for SWP images $(\times$ and $*)\left(\left(R_{e} / R_{*}\right)_{1}\right)$ and $\left(R_{e} / R_{*}\right)_{2}$ from Table 9). On top: radial velocity curve taken from Koubský et al. (1989).

Table 8. Mean values of $\left(R_{e} / R_{*}\right)$ for Fe II lines in the UV region (LWR-LWP images).

\begin{tabular}{llll}
\hline \hline Image & Date & $\left(R_{e} / R_{*}\right)$ & Phase \\
\hline LWR11064 & $15 / 07 / 81$ & 2.8 & 0.12 \\
LWR13827 & $01 / 08 / 82$ & 3.0 & 0.89 \\
LWP16693 & $29 / 10 / 89$ & 4.4 & 0.21 \\
LWP17843 & $01 / 05 / 90$ & 3.1 & 0.07 \\
LWP17905 & $15 / 05 / 90$ & 3.0 & 0.13 \\
LWP23361 & $23 / 06 / 92$ & 2.1 & 0.72 \\
\hline
\end{tabular}

Note: $\left(R_{e} / R_{*}\right)$ is the average value for multiplets (62), (63) and (64), having an excitation energy $E_{l} \approx 1 \mathrm{eV}$. The last column shows the phase calculated with the period 214.756 d estimated by Koubský et al. (1989).

Unfortunately, the lack of a good temporally dispersed set of IUE SWP-images does not allow us to cover a complete cycle of variation. However, the variations show the same tendency observed in Fig. 5, where the ratio $\left(R_{e} / R_{*}\right)$ increases between phases 0.0 and 0.2 and between phases 0.6 and 1.0.

The above results are in agreement with the observations reported by Iliev (1994), who stated that the changes of the central depths of the metal shell lines are well pronounced and show dependance on the orbital phase. The maximum and minimum values of $\left(R_{e} / R_{*}\right)$ in Fig. 5 correspond approximately to the zero radial velocity, while at the quadrature phases the envelope presents the same extent. These $\left(R_{e} / R_{*}\right)$ variations should not be interpreted as variations due to changes in the envelope size, like radial pulsation modes. Instead, according to our model, this ratio $\left(R_{e} / R_{*}\right)$ represents a mean effective distance to an equivalent ellipsoidal layer that contributes mostly to the line formation process. Under this hypothesis, the observed variations in $\left(R_{e} / R_{*}\right)$ may be attributed to the presence 
Table 9. Calculated values of $\left(R_{e} / R_{*}\right)$ for Fe II lines in the UV region (SWP images) averaged for multiplets with similar lower excitation energy.

\begin{tabular}{lllllll}
\hline \hline Image & Date & $\left(\frac{R_{e}}{R_{*}}\right)_{1}$ & $\left(\frac{R_{e}}{R_{*}}\right)_{2}$ & $\left(\frac{R_{e}}{R_{*}}\right)_{3}$ & $\left(\frac{R_{e}}{R_{*}}\right)_{4}$ & Phase \\
\hline SWP14478 & $15 / 07 / 81$ & 26.61 & 20.01 & 9.06 & 7.58 & 0.12 \\
SWP17750 & $01 / 08 / 82$ & 30.85 & 22.05 & 9.36 & - & 0.89 \\
SWP21152 & $24 / 09 / 93$ & 34.18 & 23.25 & - & - & 0.84 \\
SWP37481 & $23 / 10 / 89$ & 35.52 & 25.56 & 10.39 & 9.38 & 0.21 \\
SWP44983 & $23 / 06 / 92$ & 23.66 & 14.15 & 8.12 & 6.23 & 0.72 \\
\hline
\end{tabular}

Note: $\left(\frac{R_{e}}{R_{*}}\right)_{1}$ average ratio for multiplets $\mathrm{UV}(8), \operatorname{UV}(9), \operatorname{UV}(38)$, $\mathrm{UV}(40), \mathrm{UV}(41), \mathrm{UV}(43)$ and $\mathrm{UV}(44)\left(E_{l} \approx 0-0.4 \mathrm{eV}\right) ;\left(\frac{R_{e}}{R_{*}}\right)_{2}$ average for $\mathrm{UV}(65)$ and $\mathrm{UV}(68)\left(E_{l} \approx 1 \mathrm{eV}\right) ;\left(\frac{R_{e}}{R_{*}}\right)_{3}$ average for $\mathrm{UV}(84)$, $\mathrm{UV}(85)$ and $\mathrm{UV}(99),\left(E_{l} \approx 1.8 \mathrm{eV}\right) ;\left(\frac{R_{e}}{R_{*}}\right)_{4}$ average for $\mathrm{UV}(142)$ and $\left.\mathrm{UV}(191) E_{l} \approx 2.7 \mathrm{eV}\right)$. The last column shows the phase calculated with the period 214.756 d estimated by Koubský et al. (1989).

of an elongated extended envelope, which presents its maximum extension towards the secondary star of the binary system, and its minimun extension in the opposite direction.

The above result indicates that the envelope is strongly distorted by the gravitational field of the companion and revolves with the orbital period of $214.756 \mathrm{~d}$.

The presence of this dense elongated envelope is revealed mainly through Fe II lines. $\mathrm{H}$ lines seem to exhibit minor effects; Iliev (1994) has reported changes in the slope of the Balmer progression and in the asymmetries of $\Delta \lambda$ observed in the peaks of the emission lines. Both variations are found to correlate with the orbital motion of $214.756 \mathrm{~d}$.

This dense elongated envelope does not account for the $V / R$ variation (of $\sim 6.8$ years, see Sect. 4.3 ) observed in the Balmer lines, as it has been proposed by McLaughlin (1962) and Huang (1973) for cyclic $V / R$ variable Be stars.

It is difficult to assess the stability of an elongated envelope distorted by tidal forces. However, its existence seems possible. Evidence for the presence of this kind of asymmetric envelope was reported for Pleione (Gies et al. 1990), through $\mathrm{H} \alpha$ spectroscopy during a lunar occultation. An envelope of a mean radius of 34 to 55 stellar radii was estimated for this star and the asymmetry of the envelope was attributted to the presence of a companion, detected by speckle interferometry. Hydrodynamical models for binary stars (Gawryszczak et al. 2003) indicate that the wind from a red giant can be significantly deflected toward the orbital plane by the gravitational pull of the companion star, although these models were only developed for symbiotic stars.

The interpretation of the $\mathrm{H}$ line variations seems to be more complex. We propose that the $V / R$ variations originate as a consequence of changes in the initial velocity structure of the stellar wind, due to cyclic variations of the dynamical mechanisms driving the wind.

According to Cidale \& Ringuelet (1993), the shape of the $\mathrm{H} \alpha$ line is defined by the velocity structure. Considering a lowspeed spherical flow whose characteristics yield $\mathrm{H} \alpha$ symmetric double peak emission, then a slight increase of the mass loss would be associated with larger gradients in the velocity field at the base of the wind. It is expected that, as the wind accelerates, a flow element will see inwards and outwards photons systematically red-shifted, and therefore, the resulting profile will display a red emission enhancement while the central absorption shifts to the blue. This situation is qualitatively represented by theoretical calculations of line profiles in monotonically increasing spherical winds (see Figs. $4 \mathrm{a}$ and b in Cidale \& Ringuelet 1993). Conversely, a temporal deceleration in the wind structure might be related to a monotonically decreasing velocity case, where photons would be systematically blueshifted and interact with the blue edge of the emission profile.

Periodic long-term mass loss episodes would determine changes in the velocity and density conditions of the flow which would be related to the $V / R$ variations observed in the lines and to the long-term photometric variations as well.

\section{Conclusions}

In the present paper we have applied a semi-empirical method to obtain information about the circumstellar structure of the binary V923 Aql and have proposed a model for the envelope consistent with the observed spectroscopic variabilities.

The spectra of V923 Aql show photometric variability on periods of 0.85 day and of 6.5 years. Fe II and $\mathrm{H} \alpha$ profiles present variations with the orbital period of 214.7 days and with a quasiperiod of 6.8 years.

The analysis of the Fe II lines from 1980 to 1992 permits us to outline a distance scale for the line-forming regions. The location of these regions depends on whether the source function of the line is controlled by collisions or by photoionization. Among the transitions dominated by photoionization, the excitation energy of the lower level determines different regions of line-formation. The lines with the same lower excitation energy that are dominated by collisions form closer to the star than those dominated by photoionization. Among the multiplets dominated by the radiation field, the lines with the lowest excitation energies form further out.

The $\left(R_{e} / R_{*}\right)$ time variations reflect the influence of the secondary star over the circumstellar envelope of the primary star, the outer regions being the ones more affected. The elongated envelope has its maximum extension in the direction of the secondary and the minimum extension in the opposite direction and rotates with the orbital period. However, the revolution of an elongated envelope does not account for the observed $V / R$ variability.

Finally, taking into account long-term $V / R$ variations and Fe II line profile variations we propose that the circumstellar envelope of V923 Aquilae is consistent with an aspherical wind structure distorted by rotation and tidal forces leading to an eccentric high density envelope at the equatorial plane. Furthermore, the wind presents long-term radial velocity variations due to variations in the velocity structure, possibly modulated by a variable weak magnetic field or instabilities of the wind. 
Variations in the envelope size of V923 Aquilae, similar to the ones observed in $\gamma$ Cas (Quirrenbach et al. 1993), could be supported by interferometric observations.

The simple method presented here to study the V923 Aql extended envelope could be applied to other mild interacting binaries (e.g. $\zeta$ Tau), to study density distributions of the wind, modified by the gravitational field of the secondary component.

Acknowledgements. Part of this research was based on INES data from the IUE satellite. The authors acknowledge use of the CCD and data aquisition system supported under US National Science Foundation grant AST-90-15827 to R. M. Rich. We acknowledge J. Sahade and R. Barbá for having taken three spectra for us and C. Quiroga for his useful comments on the period analysis of $V / R$ variations.

\section{References}

Arias, M. L., Cidale, L. S., \& Ringuelet, A. E. 2000, in IAU Coll. 175, The Be Phenomenon in Early-Type Stars, ASP Conf. Ser., 214, 547, ed. M. Smith, \& H. Henrichs

Baade, D. 1987, in The Physics of Be Stars, ed. A. Slettebak, \& T. P. Snow (Cambridge: Cambridge University Press), 361

Ballereau, D., Alvarez, M., Chauville, J., \& Michel, R. 1987, Rev. Mex. Astron. Astrofis., 15, 29

Bidelman, W. P. 1950, PASP, 62, 48

Chalonge, D., \& Divan, L. 1952, Ann. Astrophys., 15, 201

Cidale, L. S. 1998, ApJ, 502, 824

Cidale, L. S., \& Ringuelet, A. E. 1989, PASP, 101, 417

Cidale, L. S., \& Ringuelet, A. E. 1993, ApJ, 411, 874

Cruzado, A. 1994, priv. comm.

Denizman, L., Koktay, T., Saygac, T., \& Kocer, D. 1994, Ap\&SS, 222, 191

Doazan, V., Sedmak, G., Barylak, M., \& Rusconi, L. 1991, in A Be atlas of far UV and optical high-resolution spectra, ESA SP-1147, 311

Dougherty, S. M., \& Taylor, A. R. 1994, MNRAS, 269, 1123

Fontaine, G., Villeneuve, B., Landstreet, J. D., \& Taylor, R. H. 1982, ApJS, 49, 259

Gayley, K. G., Ignace, R., \& Owocki, S. P. 2001, ApJ, 558, 802
Gawryszczak, A. J., Mikolajewska, J., \& Różyczka, M. 2003, A\&A, 398, 159

Gies, D. R., McKibben, W. P., Kelton, P. W., Opal, C. B., \& Sawyer, S. 1990, AJ, 100, 1601

Hanuschik, R. W., Hummel, W., Sutorius, E., et al. 1996, A\&AS, 116, 309

Harper, W. E. 1937, Pub. Dominion Astrophys. Obs., 7, 1

Horne, J. H., \& Baliunas, S. L. 1986, ApJ, 302, 757

Huang, S.-S. 1973, ApJ, 183, 541

Iliev, L. 1994, in Pulsation, rotation and mass loss in early type stars, ed. J. R. Percy, IAU Symp., 162, 374

Jefferies, J. T. 1968, Spectral Line Formation (Blaisdell Publishing and Company)

Koubský, P., Gulliver, A. F., Harmanec, P., et al. 1989, Bull. Astron. Inst. Czechosl., 40, 31

Kříž, S., \& Harmanec, P. 1975, Bull. Astron. Inst. Czechosl., 26, 65

Kurucz, R. L. 1979, ApJS, 40, 1

Kurucz, R. L. 1981, Spec. Rep. Smithsonian Ap. Obs., 390

Lynds, C. R. 1960, ApJ, 131, 390

McLaughlin, D. B. 1962, ApJS, 7, 65

Mennickent, R. E., Vogt, N., \& Sterken, C. 1994, A\&AS, 108, 237

Mennickent, R. E., Sterken, C., \& Vogt, N. 1997, A\&A, 326, 1167

Merrill, P. W. 1952, ApJ, 116, 501

Merrill, P. W., \& Lowen, L. A. 1953, ApJ, 118, 18

Mihalas, D. 1978, Stellar Atmospheres, 2nd edition (Freeman and Co.)

Moujtahid, A., Zorec, J., \& Hubert, A. M. 1998, A\&AS, 129, 289

Moujtahid, A., Zorec, J., \& Hubert, A. M. 1999, A\&A, 349, 151

Percy, J. R. 1987, in The Physics of Be Stars, ed. A. Slettebak, \& T. P. Snow (Cambridge: Cambridge University Press), 361

Percy, J. R., Coffin, B. L., Drukier, G. A., et al. 1988, PASP, 100, 1555

Pavlosvski, K., Harmanec, P., Božić, H., et al. 1997, A\&AS, 125, 75

Quirrenbach, A., Hummel, C. A., Buscher, D. F., et al. 1993, ApJ, 416, L25

Ringuelet, A. E., \& Sahade, J. 1981, PASP, 93, 594

Ringuelet, A. E., Sahade, J., Rovira, M., Fontenla, J. M., \& Kondo, Y. 1984, A\&A, 131, 9

Wiese, W. L. 1985, in Atomic Data for Controlled Fusion Research, Vol. IV, Spectroscopic Data for Iron, NBS, Washington, DC

Wiese, W. L., \& Martin, G. A. 1980, in Wavelengths and Transition Probabilities for Atoms and Atomic Ions, Part II, Washington, DC, US Government Printing Office, NSRDS-NBS, 68 